\title{
Evaluation of Genomic Selection for Seven Economic Traits in Yellow Drum (Nibea albiflora)
}

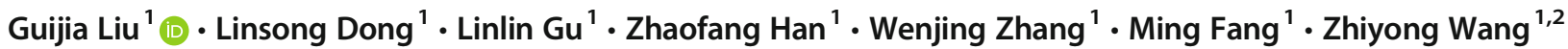

Received: 5 June 2019 / Accepted: 25 September 2019/Published online: 20 November 2019

(C) The Author(s) 2019

\begin{abstract}
Yellow drum (Nibea albiflora) is an important maricultural fish in China, and genetic improvement is necessary for this species. This research evaluated the application of genomic selection methods to predict the genetic values of seven economic traits for yellow drum. Using genome-wide single-nucleotide polymorphisms (SNPs), we estimated the genetic parameters for seven traits, including body length (BL), swimming bladder index (SBI), swimming bladder weight (SBW), body thickness (BT), body height (BH), body length/body height ratio (LHR), and gonad weight index (GWI). The heritability estimates ranged from 0.309 to 0.843 . We evaluated the prediction performance of various statistical methods, and no one method provided the highest predictive ability for all traits. We then evaluated and compared the use of genome-wide association study (GWAS)-informative SNPs and random SNPs for prediction and found that GWAS-informative SNPs obviously increased. It only needed 5 and 100 informative SNPs for LHR and BT to achieve almost the same predictive abilities as using genome-wide SNPs, and for BL, SBI, SBW, BH, and GWI, about 1000 to 3000 informative SNPs were needed to achieve whole-genome level predictive abilities. It can be concluded from the test results that breeders can use fewer SNPs to save the breeding costs of genomic selection for some traits.
\end{abstract}

Keywords Genomic selection $\cdot$ Nibea albiflora $\cdot$ Predictive ability $\cdot$ GBLUP $\cdot$ Low-density SNPs

\section{Introduction}

Genomic selection (GS) uses genome-wide single-nucleotide polymorphisms (SNPs) to obtain breeding value estimates, which was first proposed by Meuwissen et al. (2001). The key feature of the GS is that it can provide more accurate estimates for kinship among individuals compared with the

Electronic supplementary material The online version of this article (https://doi.org/10.1007/s10126-019-09925-7) contains supplementary material, which is available to authorized users.

Ming Fang

fangming618@126.com

Zhiyong Wang

zywang@jmu.edu.cn

1 Key Laboratory of Healthy Mariculture for the East China Sea, Ministry of Agriculture and Rural Affairs, Jimei University, Xiamen, China

2 Laboratory for Marine Fisheries Science and Food Production Processes, Qingdao National Laboratory for Marine Science and Technology, Qingdao, China use of pedigrees (Muir 2007). In recent years, GS has been widely carried out in livestock breeding and has made great progress, especially for dairy cattle. It usually gives higher accuracy for breeding value estimates than the best linear unbiased prediction (BLUP), but the utilization of genomic selection in aquatic animals is very few (Hayes et al. 2009; Yue 2014; Dong et al. 2016a; Dou et al. 2016). The accurate selection for aquatic animals is also important due to the fact that one pair of aquatic animals usually produces a large number of fry and will have a big economic effect (Yue 2014). To date, genomic selection has already been applied to genetically improve the economic performance of many aquatic animals, such as Atlantic salmon (Salmo salar) (Tsai et al. 2015), sea bream (Sparus aurata) (Palaiokostas et al. 2016), Yesso scallops (Patinopecten yessoensis) (Dou et al. 2016), large yellow croaker (Larimichthys crocea) (Dong et al. 2016a), Pacific white shrimp (Litopenaeus vannamei) (Wang et al. 2017), the common carp (Cyprinus carpio) (Palaiokostas et al. 2018), Japanese flounder (Paralichthys olivaceus) (Liu et al. 2018), and so on.

Yellow drum (Nibea albiflora) is an economically important marine fish in China (Sun et al. 2018). Due to overfishing 
and environmental pollution, the number of wild populations has been dropped sharply (Han et al. 2008), and the Chinese government and breeders are now paying more attention to artificial breeding of yellow drum (Yang et al. 2013; Chen et al. 2017). Yellow drum is simply selected using phenotypic selection based on body size every year. Therefore, genetic progress has been quite limited. Furthermore, some economic traits cannot be measured without killing the fish, such as eviscerated weight and meat quality. Using genomic selection not only shortens the breeding cycle but also accelerates the genetic gain (Zenger et al. 2018). With the decrease in sequencing costs, it is easy to genotype genome-wide SNPs using whole-genome sequencing or genotyping-bysequencing (GBS) (Rustagi et al. 2017). The genome of yellow drum is only approximately $565 \mathrm{Mb}$ (Han et al. 2019), which is a great advantage for use with sequencing techniques to obtain a large number of genome-wide SNPs and favors the application of genomic selection to the yellow drum genetic breeding program. Genome-wide association study (GWAS) analysis is an efficient approach for screening trait-related markers or genes (Wang et al. 2019; Yu et al. 2019). In the future, we will further conduct GWAS analysis on these traits of yellow drum to further study the genetic mechanism of these traits.

In this research, we evaluate the application of genomic selection to the genetic improvement of seven yellow drum traits: body length (BL), swimming bladder index (SBI), swimming bladder weight (SBW), body thickness (BT), body height (BH), body length/body height ratio (LHR), and gonad weight index (GWI). The GBS technique was applied to genotype genome-wide SNPs in yellow drum, estimate genetic parameters for the above seven traits, and evaluate the predictive abilities of various genomic selection strategies.

\section{Materials and Methods}

\section{Ethics Statement}

All the yellow drums came from a fish breeding company Jinling Aquaculture Science and Technology Co. Ltd. in Ningde City, Fujian Province, P.R. China. This study was approved by the Animal Care and Use Committee of the Fisheries College of Jimei University.

\section{Sample Collection and Phenotype Measurement}

In the spring of 2015, the parent fish were randomly selected from the Ningde Dongwuyang Bay. The parent fish were simultaneously injected with luteinizing hormone releasing hormone $\mathrm{A} 3$, and after 60 days of indoor breeding, the offspring were transferred to a floating cage for 1 year. Then, more than 6000 fish were randomly selected from the floating cage and transferred to the aquaculture farm. After 10 months of indoor rearing, a total of 393 yellow drums (195 males, 198 females) were collected as the experimental materials.

Severn traits were investigated, including BL, SBI, SBW, $\mathrm{BT}, \mathrm{BH}$, and GWI, which were measured in the Key Laboratory of Healthy Maricultural for the East China Sea. The weight of swimming bladder, gonads, and the body weight were measured using electronic scale with a precision of $0.01 \mathrm{~g}$; the BL, BT, and BH were directly measured using vernier caliper with precision of $0.01 \mathrm{~mm}$ (for $\mathrm{BT}$ and $\mathrm{BH}$ ) or $1 \mathrm{~mm}$ (for BL). Where SBI is the ratio of swimming bladder weight to body weight, LHR is the ratio of $\mathrm{BL}$ to $\mathrm{BH}$, and GWI is the ratio of gonad weight to body weight.

\section{Genotyping and Quality Control}

The fins of 393 fish were collected and the DNA was extracted with TIANamp Genomic DNA Kit (TIANGEN, Beijing, China); and DNA was sequenced with GBS technique implemented via Illumina HiSeq X Ten platform (Illumina, USA). After removing 22 samples with low coverage $\left(<3 \times 10^{6}\right)$, 371 samples (183 males and 188 females) were retained for SNP genotyping. The raw sequencing reads were filtered with FastQC software (Andrews 2010) and aligned to the reference genome (Han et al. 2019) using BWA v0.7.17software (Li and Durbin 2009); then, the SNPs were called with Platypusv0.8.1 (Rimmer et al. 2014), resulting in 3,868,328 SNPs. The SNPs were further filtered using plink v1.9 (Chang et al. 2015) with parameters " -vcf merge_pass.vcf - maf 0.01-geno 0.1 -hwe 1e-5-recode vcf-iid -biallelic-only -out snp -allow-extra-chr -threads 30", resulting in 53,677 SNPs with the average missing rate $3.32 \%$. The missing SNPs were imputed with software Beagle v4.1 (Browning and Browning 2016).

\section{Statistical Methods}

All phenotypes are based on a linear model:

$\mathbf{y}=\mathbf{X} \boldsymbol{\alpha}+\mathbf{B} \beta+\mathbf{e}$,

where $\mathbf{y}$ is the vector of the phenotypes for different traits; $\mathbf{X}$ is a design matrix for fixed effects; $\alpha$ is a vector of fixed effect (only the sex effect is included here); $\mathbf{B}$ is a design matrix for SNP effects (the elements in the SNPs genotypes are " 0 ," " 1 ," and "2" for genotypes "AA," "Aa," and "aa," respectively); $\beta$ is the vector for SNP effects; and $\mathbf{e}$ is the vector for residual effects. The residual effects are generally considered to be independent of each other and subject to the same distribution, $\mathbf{e} \sim N\left(0, \mathbf{I} \sigma_{e}{ }^{2}\right)$, where $\mathbf{I}$ is a vector of identity matrix and $\sigma_{e}^{2}$ is a residual variance. Genomic best linear unbiased prediction (GBLUP) (Vanraden 2008) directly predicted the genomic breeding values (GEBVs) of all individuals, and the mixed model equation can be expressed as 


$$
\left[\begin{array}{ll}
\mathbf{X}^{\prime} \mathbf{X} & \mathbf{X}^{\prime} \mathbf{Z} \\
\mathbf{Z}^{\prime} \mathbf{X} & \mathbf{Z}^{\prime} \mathbf{Z}+\mathbf{G}^{-1} \lambda
\end{array}\right]\left[\begin{array}{l}
\hat{\alpha} \\
\hat{\mathbf{g}}
\end{array}\right]=\left[\begin{array}{l}
\mathbf{X}^{\prime} \mathbf{y} \\
\mathbf{Z}^{\prime} \mathbf{y}
\end{array}\right],
$$

where $\lambda=\sigma_{e}{ }^{2} / \sigma_{g}{ }^{2}=\left(1-h^{2}\right) / h^{2} ; \sigma_{g}{ }^{2}$ is the additive genetic variance of traits; $h^{2}$ is the heritability of different traits, estimated from restrict-maximum likelihood (REML) method; and $\mathbf{Z}$ is the design matrix related to $\mathbf{g}$. The $\mathbf{G}$ matrix can be obtained from all SNP genotypes:

$\mathbf{G}=\frac{(\mathbf{B}-2 \mathbf{P})(\mathbf{B}-2 \mathbf{P})^{\prime}}{2 \sum p_{j}\left(1-p_{j}\right)}$,

where $\mathbf{P}$ is the vector of the allele frequency of each SNP and $p_{j}$ is the frequency of allele "a" at the $j$ th locus of each SNP.

We investigated 7 models for GS, BayesA (Meuwissen et al. 2001), BayesB (Meuwissen et al. 2001; Cheng et al. 2015), BayesC $\pi$ (Habier 2011), MMixp (Dong et al. 2017), ridge regression best linear unbiased prediction (RRBLUP) (Meuwissen et al. 2001), GBLUP (Vanraden 2008), and modified convolutional neural network (CNN) of Ma et al. (2018), where the CNN was modified to fit a large number of SNPs (unpublished method). In BayesB (Meuwissen et al. 2001; Cheng et al. 2015), the hyperparameter $\pi$ (the probability of including an SNP in the model) is set as 0.001, 0.01, 0.1, and $v$ (the degree of freedom of the inverse chi-square distribution) is set as 4.2 in this study. The scale parameter $\left(s^{2}\right)$ is derived by the expectation formula for the inverse chi-square distribution and $\sigma_{g}{ }^{2}$. In the GBLUP and RRBLUP methods, the variance of all SNP effects is equal in the prior distribution (Meuwissen et al. 2001; Vanraden 2008). In BayesA, all SNPs have effects, and the variances of the SNPs follow inverse chi-square distribution (Meuwissen et al. 2001). MMixp also assumes different SNPs have different variance (large variance or small variance) (Dong et al. 2017). In BayesB and BayesC $\pi$, only a small fraction of SNPs have non-zero effects (Meuwissen et al. 2001; Habier 2011; Cheng et al. 2015). The main difference between BayesB and BayesC $\pi$ is that the latter estimates the probability of the inclusion of a QTL into the model from Gibbs sampling but the former sets this parameter beforehand (Habier 2011; Cheng et al. 2015).

\section{Cross Validations}

The cross-validation was used to test prediction accuracy. The 371 individuals were randomly divided into 2 groups (reference population and testing population) with the ratio about $5: 1$, resulting in a reference population containing $\sim 334$ fish and a testing population of $\sim 37$ fish. The populations were randomly sampled for 100 times, and for each sampling we evaluated the predictive ability for each strategy, where the predictive ability was calculated as the correlation coefficient between GEBVs and phenotypes.

\section{Results}

\section{Genetic Parameter Estimates}

The statistical results of phenotypic data of 7 traits are shown in Table 1. We tested the gender effect by comparing the phenotypes between the male and female group using $t$ test for 7 traits. As shown in Table 1, gender has significant effect on the BL, GWI, SBI, BT, and BH, but not on the LHR and SBW. The phenotypes for the males and females of the sample sets are summarized in Table 1 . There are significant differences for BL, SBI, BT, BH, and GWI between genders, suggesting that a gender effect must be considered in the estimation of breeding values. The variance components $\left(\sigma_{g}{ }^{2}\right.$ and $\left.\sigma_{e}^{2}\right)$ and heritability $\left(h^{2}\right)$ with 371 individuals were estimated using GVCBLUP software (Wang et al. 2014). The results are also presented in Table 1 . The heritability of LHR was relatively lower $(0.309 \pm 0.140)$ compared with the other traits, and BL has the highest heritability $(0.843 \pm 0.150)$ among the 7 traits investigated (we will explain the overestimate of it in discussion). The heritability estimates of 6 of the 7 traits exceeded 0.4 , suggesting these traits had the potentiality to be genetically improved.

\section{Performance of Different GS Methods}

The predictive abilities for 7 traits are shown in Table 2. It was also noted that in BayesB (Meuwissen et al. 2001; Cheng et al. 2015), the hyperparameters have a great effect on prediction accuracy, and we chose the best one here. Using different algorithms, the predictive abilities ranged from 0.361 to 0.396 for BL, from 0.145 to 0.207 for LHR, from 0.203 to 0.242 for SBI, from 0.180 to 0.238 for SBW, from 0.283 to 0.317 for $\mathrm{BT}$, from 0.350 to 0.400 for $\mathrm{BH}$, and from 0.374 to 0.412 for GWI. The maximum difference and percentages of the differences across algorithms are also listed in Table 2. For some traits, such as BL and GWI, the difference among methods is small $(<10 \%)$, whereas for the other 5 traits, the differences were more than $10 \%$, and as even as high as $24.4 \%$. However, none of the methods consistently provided the highest predictive ability for all the traits. Among these traits, $\mathrm{BL}, \mathrm{BH}$, and GWI have higher prediction accuracy than the other traits.

\section{Prediction Performance with Different Numbers of SNP Sets}

To reduce the cost of selection, we evaluated the performance of genomic selection with an SNP set rather than using wholegenome SNPs. We built the SNP set in two ways. The first method was based on informative SNPs that were selected according to $p$ values (from small to big) of GWAS that implemented via EMMAX software (Perry 2000; Meuwissen 
Table 1 Statistical results for phenotypic data for seven traits

\begin{tabular}{|c|c|c|c|c|c|c|c|}
\hline \multirow[t]{2}{*}{ Trait } & \multicolumn{2}{|l|}{ Male } & \multicolumn{2}{|l|}{ Female } & \multicolumn{3}{|l|}{371 individuals } \\
\hline & Number & Mean \pm S.D. & Number & Mean \pm S.D. & $\sigma_{g}^{2} \pm$ S.E. & $\sigma_{e}^{2} \pm$ S.E. & $h^{2} \pm$ S.E. \\
\hline $\mathrm{BL}$ & 183 & $211.80 \pm 15.45 \mathrm{~mm}^{* *}$ & 188 & $221.46 \pm 16.08$ & $237.123 \pm 59.328$ & $44.210 \pm 39.475$ & $0.843 \pm 0.150$ \\
\hline LHR & 183 & $2.99 \pm 0.21(-)$ & 188 & $2.99 \pm 0.22$ & $0.015 \pm 0.007$ & $0.032 \pm 0.006$ & $0.309 \pm 0.140$ \\
\hline SBI & 183 & $0.72 \pm 0.30 \% *$ & 188 & $0.62 \pm 0.42$ & $556.844 \pm 251.654$ & $830.842 \pm 200.971$ & $0.401 \pm 0.162$ \\
\hline SBW & 183 & $1.56 \pm 0.68 \mathrm{~g}(-)$ & 188 & $1.52 \pm 0.84$ & $0.301 \pm 0.120$ & $0.330 \pm 0.092$ & $0.477 \pm 0.165$ \\
\hline BT & 183 & $35.81 \pm 4.05 \mathrm{~mm} *$ & 188 & $37.30 \pm 3.98$ & $11.923 \pm 3.377$ & $5.498 \pm 2.379$ & $0.684 \pm 0.151$ \\
\hline $\mathrm{BH}$ & 183 & $71.14 \pm 6.68 \mathrm{~mm}^{*}$ & 188 & $74.42 \pm 5.95$ & $29.656 \pm 7.939$ & $12.449 \pm 5.540$ & $0.704 \pm 0.145$ \\
\hline GWI & 183 & $1.11 \pm 0.43 \% * *$ & 188 & $0.91 \pm 0.25$ & $0.104 \pm 0.025$ & $0.030 \pm 0.017$ & $0.773 \pm 0.140$ \\
\hline
\end{tabular}

*, ** Significant differences between sex at the 0.01 and 0.001 levels, respectively

et al. 2001; Kang et al. 2010). The different number of SNPs were $5,10,15,20,30,100,200,300,400,1000,2000,3000$, $5000,10,000,20,000,30,000,40,000$, and 53,677 (all). In contrast, the second method chose the same number of SNP set randomly. The GBLUP method (Vanraden 2008) was used to estimate the genetic values, and cross-validation was employed to test the predictive ability.

As shown in Fig. 1, generally, the predictive abilities were relatively higher with an informative SNP set than with a random SNP set, which suggests that informative SNPs are helpful in increasing predictive abilities; furthermore, for both strategies, the predictive abilities improved with an increase in the number of SNPs. Using 5 random SNPs, the predictive abilities were very close to zero or below zero for all traits, and compared with this, with the use of 5 informative SNPs, it produces more obvious predictive abilities for some of the traits, such as LHR, BT, and GWI. It is also shown in Fig. 1 that for BL, SBI, SBW, BH, and GWI, a set of 1000 to 3000 informative SNPs could bring almost the same predictive abilities as using the whole-genome SNPs; and for BT and LHR, it only needs approximately 100 and 5 informative SNP to achieve the same predictive abilities as using whole-genome SNPs. However, random SNP set requires much more SNPs (typically tens of thousands) to achieve similar predictive abilities to those achieved by using whole-genome SNPs. The results of LHR in Fig. 1b decreased from 30 to 2000 SNPs. The reason is that the sample size is small, and the pedigree of this population is complex, making the results a bit unstable, but which does not affect to get the general trend.

\section{Discussion}

This research evaluated the performance of using genomic selection to predict breeding values for yellow drum, which provides a reference for future breeding programs. The study also successfully estimated the genetic parameters for 7 traits using genome-wide SNPs, which provides direct and accurate information to build kinship among individuals, and thus leads to accurate estimates for genetic parameters. Though heritability is usually estimated with pedigree information, by which the kinship among individuals can be built, it is very

Table 2 Effect of different methods on the predictive ability of 7 traits

\begin{tabular}{|c|c|c|c|c|c|c|c|c|}
\hline \multirow[t]{2}{*}{ Methods } & \multirow[t]{2}{*}{ Parameter } & \multicolumn{7}{|c|}{ Predictive abilities (mean \pm standard errors) } \\
\hline & & $\mathrm{BL}$ & LHR & SBI & SBW & BT & $\mathrm{BH}$ & GWI \\
\hline BayesA & & $0.361 \pm 0.011$ & $0.157 \pm 0.014$ & $0.209 \pm 0.014$ & $0.184 \pm 0.013$ & $0.315 \pm 0.017$ & $0.355 \pm 0.014$ & $0.397 \pm 0.013$ \\
\hline \multirow[t]{3}{*}{ BayesB } & $\pi=0.001$ & $0.240 \pm 0.015$ & $0.186 \pm 0.014$ & $0.177 \pm 0.016$ & $0.139 \pm 0.017$ & $0.259 \pm 0.016$ & $0.363 \pm 0.012$ & $0.349 \pm 0.014$ \\
\hline & $\pi=0.01$ & $0.334 \pm 0.015$ & $0.186 \pm 0.014$ & $0.242 \pm 0.018$ & $0.191 \pm 0.016$ & $0.293 \pm 0.015$ & $0.393 \pm 0.013$ & $0.394 \pm 0.012$ \\
\hline & $\pi=0.1$ & $0.362 \pm 0.014$ & $0.191 \pm 0.014$ & $0.230 \pm 0.016$ & $0.214 \pm 0.015$ & $0.298 \pm 0.015$ & $0.397 \pm 0.013$ & $0.400 \pm 0.012$ \\
\hline \multicolumn{2}{|l|}{ BayesC $\pi$} & $0.396 \pm 0.014$ & $0.165 \pm 0.014$ & $0.203 \pm 0.013$ & $0.180 \pm 0.014$ & $0.317 \pm 0.016$ & $0.384 \pm 0.015$ & $0.391 \pm 0.012$ \\
\hline \multicolumn{2}{|l|}{ MMixp } & $0.378 \pm 0.013$ & $0.207 \pm 0.014$ & $0.215 \pm 0.013$ & $0.195 \pm 0.012$ & $0.296 \pm 0.017$ & $0.400 \pm 0.012$ & $0.386 \pm 0.014$ \\
\hline \multicolumn{2}{|l|}{ RRBLUP } & $0.380 \pm 0.015$ & $0.192 \pm 0.014$ & $0.224 \pm 0.013$ & $0.218 \pm 0.013$ & $0.298 \pm 0.015$ & $0.380 \pm 0.012$ & $0.389 \pm 0.012$ \\
\hline \multicolumn{2}{|l|}{ GBLUP } & $0.378 \pm 0.015$ & $0.174 \pm 0.013$ & $0.219 \pm 0.014$ & $0.238 \pm 0.011$ & $0.305 \pm 0.017$ & $0.365 \pm 0.013$ & $0.374 \pm 0.014$ \\
\hline \multicolumn{2}{|l|}{$\mathrm{CNN}$} & $0.392 \pm 0.012$ & $0.145 \pm 0.015$ & $0.207 \pm 0.014$ & $0.211 \pm 0.016$ & $0.283 \pm 0.015$ & $0.350 \pm 0.014$ & $0.412 \pm 0.012$ \\
\hline \multicolumn{2}{|c|}{$\begin{array}{l}\text { Maximum difference } \\
\text { (percent of the difference) }\end{array}$} & $0.035(8.8 \%)$ & $0.050(24.2 \%)$ & $0.039(16.1 \%)$ & $0.058(24.4 \%)$ & $0.034(10.7 \%)$ & $0.05(12.5 \%)$ & $0.038(9.2 \%)$ \\
\hline
\end{tabular}


a

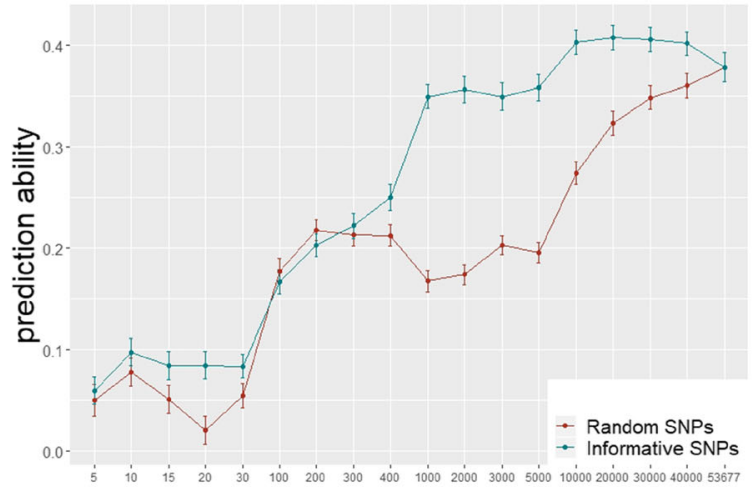

C

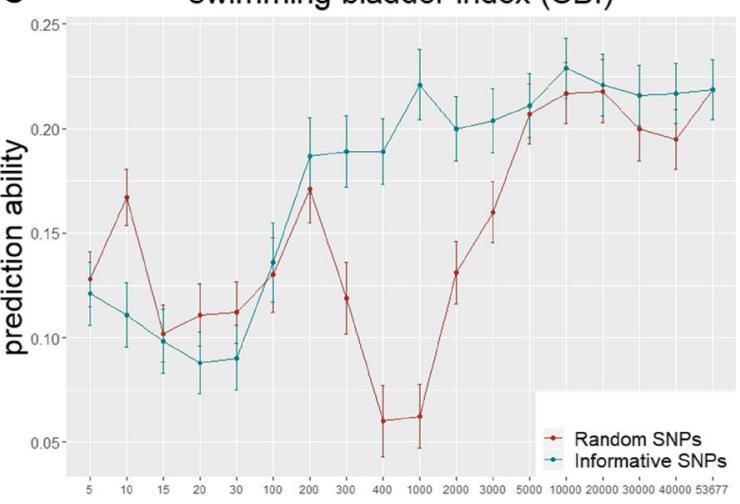

e

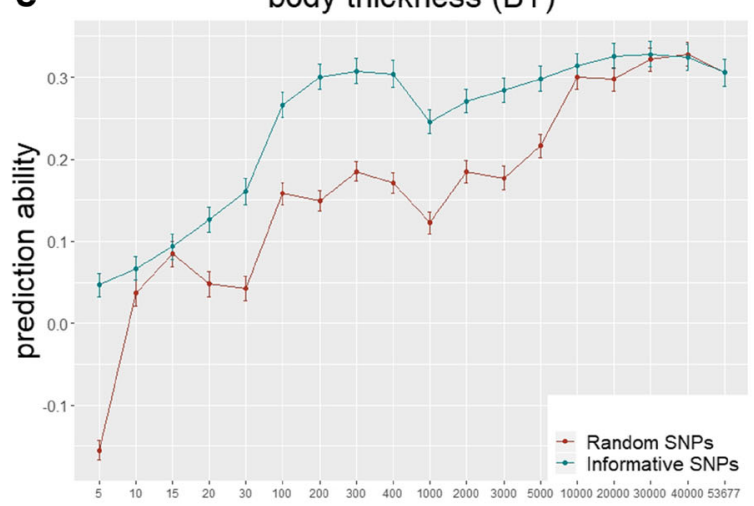

g

gonad weight index (GWI)

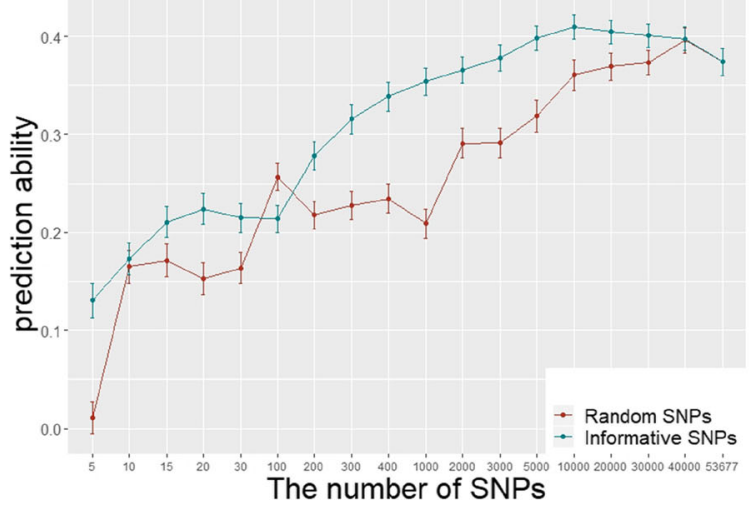

b

body length/body height ratio (LHR)

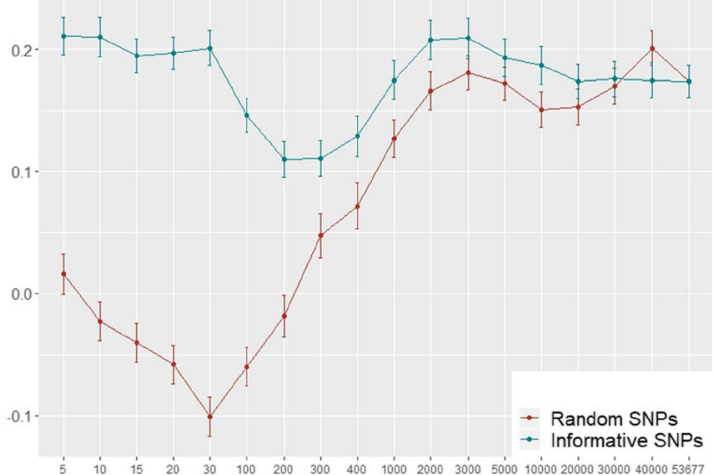

d

swimming bladder weight (SBW)

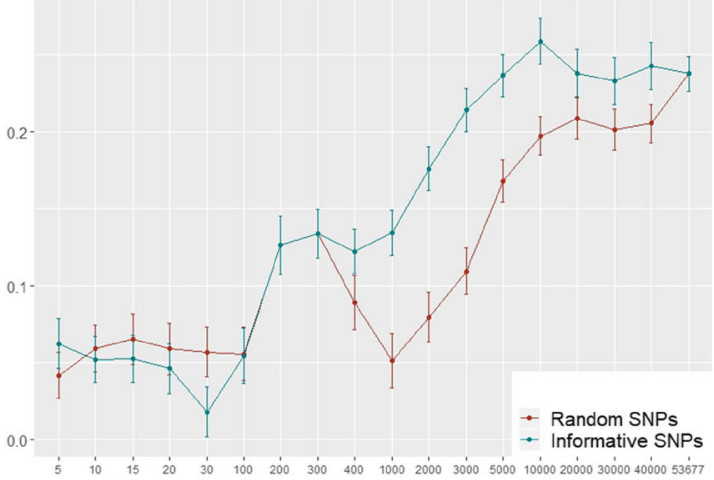

\section{f}

body height $(\mathrm{BH})$

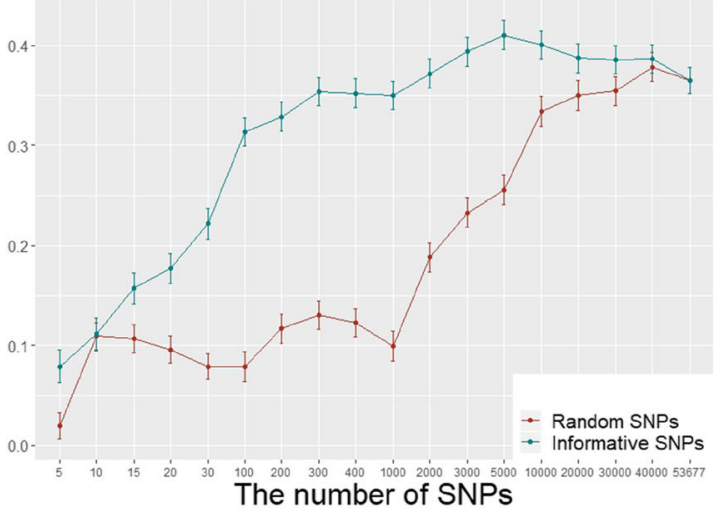

ig. 1 Predictive abilities under different numbers of GWAS informative SNPs and SNPs randomly selected by GBLUP 
hard to get for yellow drum and they are also less accurate than using SNPs. The heritability estimates of BL, BH, and GWI, are $0.843,0.704$, and 0.773 , respectively, and it seems that they are higher than expected value (0.4 0.6). Nevertheless, the $99 \%$ confidence intervals of the estimates are $(0.457,1)$, $(0.330,1)$, and $(0.412,1)$, respectively (obtained from the estimated heritability plus and minus two times of the standard error), showing that they include the expected value (0.4 0.6), suggesting the estimated heritability is within a statistically acceptable scope. In another hand, we only used a small number of fish (371) in this study and they were the offspring of several parents, maybe not representative for the whole population, and may lead to biased estimates for the heritability. The ideal population for the estimates of the heritability is a balanced population that consists as many families with similar number of offspring.

We also tested the prediction performance of different methods, including Bayesian methods, GBLUP (Vanraden 2008), and modified CNN method of Ma et al. (2018), but none always gave the highest predictive ability for all the traits. This suggests that when applying genomic selection for genetic breeding, we need to evaluate various methods and then choose the most suitable one for a specific trait (Dong et al. 2016b).

The size of the population is the most important factor affecting the predictive abilities. In this research, we only generated very small reference population ( 334 fish), which led to low predictive abilities. The research inferred the size of the reference population using the method from Daetwyler et al. (2008). We fit the linear equation mentioned in Supplementary Table 2 of this paper and found that if we aim to achieve 0.8 of the prediction accuracy, then it needs 3773, 4206, 3621, 4606, 1640, 1702, and 2116 fish for BL, LHR, SBI, SBW, BT, BH, and GWI, respectively (see supplementary for detailed calculation). Another factor that affects the prediction ability is the genotyping methods. We used the GBS technique for SNP genotyping and generated 50,000 SNPs, but it is still not dense enough to cover causal mutations. Compared with it, whole-genome sequencing is probably able to further increase the prediction abilities. The last factor that affects the prediction accuracy is the statistical methods; we have applied seven statistical methods for genomic selection in this study, and the results showed that the performance among methods (ranging from 8.8 to $24.4 \%$ ) varied greatly. This suggests that statistical method has an ineligible effect on genomic selection. Hopefully, some sophisticated methods will be developed in the future that is able to further increase the predictive ability and suitable for more traits.

To conveniently evaluate the performance of SNP sets for genomic selection, we only investigated on the GBLUP method (Vanraden 2008). We compared the GWAS-informative SNPs to randomly selected SNPs and showed that GWAS- informative SNPs could bring predictive abilities as close as those using whole-genome SNPs, and the number of informative SNPs required ranged from 5 to 3000 depending on the traits. This conclusion is generally consistent with that of Abed et al. (2018) and Dassonneville et al. (2011), suggesting that one can use only a small number of informative SNPs rather than whole-genome SNPs for selection, which would dramatically reduce breeding costs (Habier et al. 2009; Dong et al. 2016a; Song et al. 2019).

Acknowledgments Kun Ye, Yangjie Xie, Sha Sun, and other colleagues in the laboratory participated in fish sampling and trait measurements.

Author Contributions ZW provided the data for the analysis. MF designed the study and directed the analysis. $\mathrm{ZH}$ and $\mathrm{WZ}$ provided bioinformatics support. GL conducted the analysis and interpreted the results. LG provided technical support for artificial intelligence data analysis. LD provided technical support for the Bayesian methods. GL wrote the first draft of the manuscript, and every author has reviewed the work.

Funding This work was supported by the China Agriculture Research System (CARS-47-G04) and the National Natural Science Foundation of China (No. 31672399 and 31872560).

Availability of Data Raw DNA sequencing reads were deposited in NCBI with the project accession of PRJNA533721.

\section{Compliance with Ethical Standards}

The authors declare that the research was conducted in the absence of any commercial or financial relationships that could be construed as a potential conflict of interest.

Open Access This article is distributed under the terms of the Creative Commons Attribution 4.0 International License (http:// creativecommons.org/licenses/by/4.0/), which permits unrestricted use, distribution, and reproduction in any medium, provided you give appropriate credit to the original author(s) and the source, provide a link to the Creative Commons license, and indicate if changes were made.

\section{References}

Abed A, Pérez-Rodríguez P, Crossa J, Belzile F (2018) When less can be better: How can we make genomic selection more cost-effective and accurate in barley? Theor Appl Genet 131:1-18

Andrews S (2010) FastQC: a quality control tool for high throughput sequence data, Babraham Bioinformatics. Babraham Institute, Cambridge

Browning BL, Browning SR (2016) Genotype imputation with millions of reference samples. Am J Hum Genet 98:116-126

Chang CC, Chow CC, Tellier LC, Vattikuti S, Purcell SM, Lee JJ (2015) Second-generation PLINK: rising to the challenge of larger and richer datasets. Gigascience 4:7

Chen R, Lou B, Xu D, Zhan W, Takeuchi Y, Yang F et al (2017) Induction of meiotic gynogenesis in yellow drum (Nibea albiflora, Sciaenidae) using heterologous sperm and evidence for female homogametic sex determination. Aquaculture 479:667-674 
Cheng H, Qu L, Garrick DJ, Fernando RL (2015) A fast and efficient Gibbs sampler for BayesB in whole-genome analyses. Genet Sel Evol 47:80-87

Daetwyler HD, Beatriz V, Woolliams JA (2008) Accuracy of predicting the genetic risk of disease using a genome-wide approach. PLoS One 3:e3395-e3395

Dassonneville R, Brøndum RF, Druet T, Fritz S, Guillaume F, Guldbrandtsen B, Lund MS, Ducrocq V, Su G (2011) Effect of imputing markers from a low-density chip on the reliability of genomic breeding values in Holstein populations. J Dairy Sci 94: 3679-3686

Dong L, Xiao S, Chen J, Wan L, Wang Z (2016a) Genomic selection using extreme phenotypes and pre-selection of SNPs in large yellow croaker (Larimichthys crocea). Mar Biotechnol 18:575-583

Dong L, Xiao S, Wang Q, Wang Z (2016b) Comparative analysis of the GBLUP, emBayesB, and GWAS algorithms to predict genetic values in large yellow croaker (Larimichthys crocea). BMC Genomics 17:460-411

Dong L, Fang M, Wang Z (2017) Prediction of genomic breeding values using new computing strategies for the implementation of MixP. Sci Rep 7:17200-17211

Dou J, Li X, Fu Q, Jiao W, Li Y, Li T et al (2016) Evaluation of the 2bRAD method for genomic selection in scallop breeding. Sci Rep 6: 19244

Habier D (2011) Extension of the bayesian alphabet for genomic selection. Bmc Bioinformatics 12(1):186

Habier D, Fernando RL, Dekkers JCM (2009) Genomic selection using low-density marker panels. Genetics 182:343

Han ZQ, Gao TX, Yanagimoto T, Sakurai Y (2008) Genetic population structure of Nibea albiflora in Yellow Sea and East China Sea. Fish Sci 74:544-552

Han Z, Li W, Zhu W, Sun S, Ye K, Xie Y et al (2019) Near-complete genome assembly and annotation of the yellow drum (Nibea albiflora) provide insights into population and evolutionary characteristics of this species. Ecol Evol 9:568-575

Hayes BJ, Bowman PJ, Chamberlain A, Goddard M (2009) Invited review: genomic selection in dairy cattle: progress and challenges. J Dairy Sci 92:433-443

Kang HM, Sul JH, Service SK, Zaitlen NA, Kong SY, Freimer NB et al (2010) Variance component model to account for sample structure in genome-wide association studies. Nat Genet 42:348-354

Li H, Durbin R (2009) Fast and accurate short read alignment with burrows-wheeler transform. Bioinformatics 25:1754-1760

Liu Y, Lu S, Liu F, Shao C, Zhou Q, Wang N et al (2018) Genomic selection using BayesC $\pi$ and GBLUP for resistance against Edwardsiella tarda in Japanese Flounder (Paralichthys olivaceus). Mar Biotechnol 20:559-565

Ma W, Qiu Z, Jie S, Li J, Qian C, Zhai J et al (2018) A deep convolutional neural network approach for predicting phenotypes from genotypes. Planta 248:1307-1318

Meuwissen TH, Hayes BJ, Goddard ME (2001) Prediction of total genetic value using genome-wide dense marker maps. Genetics 157: $1819-1829$

Muir W (2007) Comparison of genomic and traditional BLUP-estimated breeding value accuracy and selection response under alternative trait and genomic parameters. J Anim Breed Genet 124:342-355

Palaiokostas C, Ferraresso S, Franch R, Houston RD, Bargelloni L (2016) Genomic prediction of resistance to pasteurellosis in gilthead sea bream (Sparus aurata) using 2b-RAD sequencing. G3 (Bethesda) 6:3693-3700

Palaiokostas C, Kocour M, Prchal M, Houston RD (2018) Accuracy of genomic evaluations of juvenile growth rate in common carp (Cyprinus carpio) using genotyping by sequencing. Front Genet 9:82

Perry SF (2000) Which came first, the breath or the lung? Comp Biochem Physiol B Biochem Mol Biol 126:S77-S77

Rimmer A, Phan H, Mathieson I, Iqbal Z, Twigg SR, Wilkie AO et al (2014) Integrating mapping-, assembly-and haplotype-based approaches for calling variants in clinical sequencing applications. Nat Genet 46:912

Rustagi N, Zhou A, Watkins WS, Gedvilaite E, Wang S, Ramesh N, Muzny D, Gibbs RA, Jorde LB, Yu F, Xing J (2017) Extremely low-coverage whole genome sequencing in South Asians captures population genomics information. BMC Genomics 18:396-312

Song K, Wen S, Zhang G (2019) Adaptive evolution patterns in the pacific oyster Crassostrea gigas. Mar Biotechnol. https://doi.org/ 10.1007/s10126-019-09906-w

Sun S, Li W, Xiao S, Lin A, Han Z, Cai M et al (2018) Genetic sex identification and the potential sex determination system in the yellow drum (Nibea albiflora). Aquaculture 492:253-258

Tsai H-Y, Hamilton A, Tinch AE, Guy DR, Gharbi K, Stear MJ et al (2015) Genome wide association and genomic prediction for growth traits in juvenile farmed Atlantic salmon using a high density SNP array. BMC Genomics 16:969

Vanraden PM (2008) Efficient methods to compute genomic predictions. J Dairy Sci 91(11):4414-4423

Wang C, Prakapenka D, Wang S, Pulugurta S, Runesha HB, Da Y (2014) GVCBLUP: a computer package for genomic prediction and variance component estimation of additive and dominance effects. BMC Bioinformatics 15:270

Wang Q, Yu Y, Yuan J, Zhang X, Huang H, Li F, Xiang J (2017) Effects of marker density and population structure on the genomic prediction accuracy for growth trait in Pacific white shrimp Litopenaeus vannamei. BMC Genet 18:45-49

Wang W, Tan S, Luo J, Shi H, Zhou T, Yang Y et al (2019) GWAS analysis indicated importance of NF-kB signaling pathway in host resistance against motile Aeromonas septicemia disease in catfish. Mar Biotechnol 21:335-347

Yang Y, Xie Y, Cai M, Jian L, Chen Q, Lou B et al (2013) Induction and identification of gynogenesis in Nibea albiflora. J Fish China 37: 1297-1303

Yu Y, Wang Q, Zhang Q, Luo Z, Wang Y, Zhang X et al (2019) Genome scan for genomic regions and genes associated with growth trait in pacific white shrimp Litopeneaus vannamei. Mar Biotechnol 21: 374-383

Yue GH (2014) Recent advances of genome mapping and markerassisted selection in aquaculture. Fish Fish 15:376-396

Zenger KR, Khatkar MS, Jones DB, Khalilisamani N, Jerry DR, Raadsma HW (2018) Genomic selection in aquaculture: application, limitations and opportunities with special reference to marine shrimp and pearl oysters. Front Genet 9:693

Publisher's note Springer Nature remains neutral with regard to jurisdictional claims in published maps and institutional affiliations. 